\title{
El sistema asistencial en Pamplona a través de la geografía de sus defunciones (1880-1935)
}

\author{
Sagrario Anaut Bravo*
}

\begin{abstract}
RESUMEN
ABSTRACT

Con el análisis del lugar donde se produce la defunción de los habitantes de Pamplona (1880-1935), se ha podido confirmar que, a pesar de los cambios político-económicos de la época, el sistema asistencial seguía dentro del modelo heredado. EI desempleo, la subalimentación, el analfabetismo y la desprotección de la mayoría de la población - mujeres, ancianos y niños - serán los cuatro pilares sobre los que se asienten el nivel de salud y la evolución de la población. De esta forma, la mortalidad en Pamplona no pudo descender y modernizarse hasta los años treinta del siglo $X X$.

PALABRAS CLAVE Sistema asistencial, mortalidad, salud, inclusa.

Analysing the geographical location of the places where the inhabitants of Pamplona died, it has been confirmed that, in spite of the social-economic changes of the period, the Health Service continued within the traditional model. Unemployment, undernourishment, illiteracy and the lack of protection of the majority of the population -women, elderly people and children-- are the main elements which determine the health standard and the population development. Considering the above data, it becomes evident that, the mortality rate in Pamplona couldn't drop and that the modernisation process didn't take place.

KEY WORDS

Health service, mortality, health, foundling hospital.
\end{abstract}

- Departamento de Geografia e Historia, U.P.N.A. 


\section{INTRODUCCIÓN}

Con una propuesta metodológica poco habitual dentro de la historiografía navarra y española se va a proceder al estudio del grado de desarrollo y de la evolución seguida por el sistema benéfico-asistencial de Pamplona en un período de la historia de la asistencia social bastante delicado por ser de tránsito hacia su redefinición de objetivos y medios de actuación. Hasta el momento, las preferencias historiográficas han optado por el análisis en profundidad de las instituciones más relevantes y la legislación que sobre esta materia se fue gestando. Para el caso navarro son todavía muy escasas las aportaciones que sobre el sistema asistencial han visto la luz, y lo poco que hay sigue líneas muy dispares, no abarca un período que supere el primer tercio del siglo $x x$ o descarta ciertas instituciones como los asilos o el Hospital Militar ${ }^{1}$.

Frente a este panorama, se propone un ejercicio de reflexión basado en el conocimiento de la geografía de las defunciones en el período elegido, a partir del cual se podrá conocer el papel que cumplieron las principales instituciones asistenciales y de sanación de la capital navarra. En la medida que se pueda percibir un mayor protagonismo del Hospital Civil o de la Inclusa, también se podrá atestiguar una mejora de sus prestaciones, un nuevo interés por los grupos pauperizables y no solo por los pauperizados registrados en los Padrones de Pobres, y un lento progreso hacia la adecuación de las instituciones y servicios asistenciales a las exigencias de la nueva sociedad liberal.

\section{LA GEOGRAFIA DE LA MUERTE}

La principal fuente que informa sobre el lugar en el que se produjo el fallecimiento de un residente de Pamplona son las partidas de defunción del Registro Civil. A partir del vaciado uninominal de dichas partidas para los años 1881, 1906 y 1931 se ha podido confeccionar el cuadro 1 que sintetiza la realidad que nos proponemos analizar. Igualmente, se ha constatado la presencia de ciertos errores en el registro que cuestionan de al-

Las obras más importantes son: Ramos MaRtinez, J., La salud pública y el Hospital General de Pamplona en el Antiguo Régimen (1700-1815), Gobierno de Navarra, 1989; VALVERDE, L., Entre el deshonor y la miseria. Infancia abandonada en Guipúzcoa y Navarra, ss. xvIII y xIx, U.P.V., 1994: Uribe-Etxeberria, A., Marginalidad protegida: mujeres y niños abandonados en Navarra (1890-1930), U.P.V., 1996. Hay algunos artículos sueltos en las revistas Principe de Viana y del Instituto Gerónimo de Ustariz. 
guna manera la fiabilidad de los datos. Este problema no alcanza la categoría del que se produce en el registro de otras variables como la edad y no tiene la suficiente entidad para ser valorado en este estudio por el tipo de agrupación que se ha elegido ?.

La primera información que se desprende del cuadro 1 hace referencia al predominio absoluto, a lo largo del medio siglo elegido, de las residencias familiares como los lugares en los que se producen los fallecimientos. Esta preferencia no sólo se debe a la tradición de un sistema benéficoasistencial no enfocado a la sanación de enfermos crónicos o que requerían un período dilatado de atenciones médicas, también entrarán en juego múltiples factores difíciles de valorar como las deficiencias y el carácter estigmatizante de los centros sanitarios y asistenciales dependientes de la Diputación y del Ayuntamiento, la preferencia por una asistencia domiciliaria benéfica o privada, o como la tradición cultural cristiana que habla del cuidado de los enfermos por su familia ${ }^{3}$. Esta aparente uniformidad puede ser matízada con la desagregación de los menores de diez años respecto del total, por cuanto son un segmento de la población especialmente vulnerable a la enfermedad y la muerte.

\section{Cuadro 1: Proporcionalidad de las defunciones según el lugar} del fallecimiento $\left(1881,1906\right.$ y 1931) ${ }^{4}$

\begin{tabular}{crcrrrr}
\hline & & \multicolumn{6}{c}{1881} \\
& TOT. & $\%$ & \multicolumn{1}{c}{$V^{2}$} & $\%$ & $M$ & $\%$ \\
\hline c.asist. & 66 & 6,46 & 34 & 6,15 & 32 & 6,87 \\
c.especial & 67 & 6,56 & 62 & 11,2 & 5 & 1,07 \\
resid.famil. & 863 & 84,5 & 442 & 79,9 & 420 & 90,1 \\
s.es. & 25 & 2,45 & 15 & 2,71 & 9 & 1,93 \\
Total & 1.021 & & 553 & & 466 & \\
\hline
\end{tabular}

2 Los mayores problemas suelen producirse en el registro del número de la calle y piso, y en el nombre de la calle y en la ausencia de este dato en algunas partidas. A esta conclusión se ha podido llegar al cotejar los datos de los libros de defunciones del Registro Civil con los mismos libros del Ayuntamiento de Pamplona.

3 No hay que perder de vista que el período aquí analizado coincide con un momento en el que todavia no se ha cerrado la modernización del sistema benéfico y asistencial heredado de un pasado en el que se hallaba bajo la tutela de la !glesia. Son años donde las competencias en esta materia van pasando del Estado al municipio, de instancias religiosas a civiles, donde los objetivos no están del todo perfilados, donde la principal preocupación es la dimensión económica, etc.

${ }_{4}$ c.asist.: centros asistenciales como asilos, Inclusa, Casa de Misericordia, Maternidad, Manicomio y Hospital Civil; c.especial: conventos y Hospital Militar; resid.famil.: residencias 


\begin{tabular}{|c|c|c|c|c|c|c|c|c|}
\hline \multicolumn{9}{|c|}{1906} \\
\hline & TOT. & $\%$ & V & $\%$ & $M$ & $\%$ & S. sex & $\%$ \\
\hline c.asist. & 80 & 9,02 & 38 & 9,45 & 36 & 8,41 & 6 & 10,5 \\
\hline c.especial & 11 & 1,24 & 7 & 1,74 & 4 & 0,93 & 0 & 0 \\
\hline resid.famil. & 789 & 88,9 & 356 & 88,6 & 386 & 90,2 & 47 & 82,5 \\
\hline s.es. & 5 & 0,56 & 1 & 0,25 & 2 & 0,47 & 2 & 3,51 \\
\hline s.l. & 2 & 0,22 & & & & & 2 & 3,51 \\
\hline Total & 887 & & 402 & & 428 & & 57 & \\
\hline \multicolumn{9}{|c|}{1931} \\
\hline & TOT. & $\%$ & V & $\%$ & M & $\%$ & S. sex & $\%$ \\
\hline c.asist. & 162 & 16,3 & 91 & 17,6 & 55 & 13,5 & 16 & 22,5 \\
\hline c.especial & 3 & 0,302 & 2 & 0,4 & 1 & 0,24 & 0 & 0 \\
\hline resid.famil. & 805 & 81,06 & 418 & 81 & 349 & 86 & 38 & 53,5 \\
\hline s.es. & 23 & 2,316 & 5 & 0,97 & 1 & 0,24 & 17 & 23,9 \\
\hline Total & 993 & & 516 & & 406 & & 71 & \\
\hline
\end{tabular}

FUENTE: Elaboración propia a partir de los libros de defunción del Registro Civil de Pamplona.

En el gráfico 1 se describe un proceso parcialmente diferente al comentado con anterioridad. El gradual descenso de las defunciones registradas en las residencias familiares desde el casi $90 \%$ del total de los decesos de los menores de diez años en 1880 hasta el $55 \%$ de 1933 , quedará roto bruscamente a partir de 1934 . No hubo mejoras ni aumento del número de instituciones de asistencia y sanación dedicadas a este segmento de la población menor de diez años en estas fechas, por lo que la evolución apuntada debió estar condicionado por un cambio en el comportamiento de las madres tanto solteras como casadas que optaron por dar a luz en la Casa de Maternidad ${ }^{5}$ en lugar de en sus residencias o en

particulares en régimen de propiedad, alquiler, etc.; s.es.: sin especificarse el lugar o son ilegibles - suele darse coincidiendo con cambios de registradores-; s.l.: en la calle, en el río,...en situación de abandono.

5 El departamento de casadas de la Casa de Maternidad no fue inaugurado hasta marzo de 1929 , pero ya ingresaban con anterioridad. Igualmente, cuando el parto era complicado se acudia a) Hospital. 
otros lugares como «las casas clandestinas para dar a luz» ${ }^{6}$, muy útiles para salvaguardar el honor de la mujer. Las campañas promovidas desde el Ayuntamiento en favor de este tipo de asistencia sanitaria en el momento del parto, capaz de reducir la mortalidad entre las madres y los recién nacidos, debieron dar sus frutos, tal y como lo demuestran los ingresos recogidos por A. Uribe-Etxeberria ? Según esta autora, a partir de 1905 y hasta 1934 los ingresos en la Maternidad experimentaron un importante crecimiento que no necesariamente debió estar provocado por un aumento de la miseria o/y de nacimientos ilegítimos.

Gráfico 1: Las defunciones infantiles y juveniles de Pamplona atendiendo al lugar donde quedaron registradas (\%).

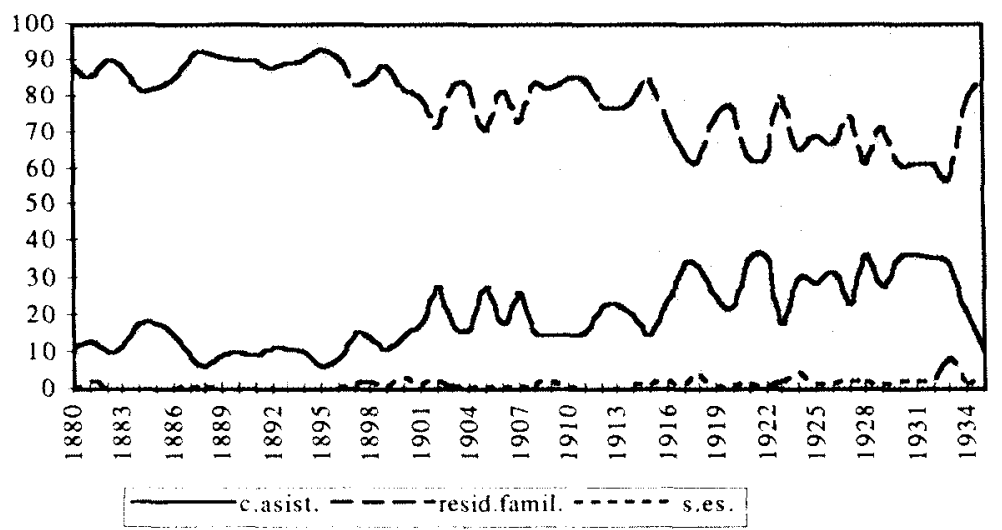

FUENTE: Elaboración propia a partir de los libros de defunciones del Registro Civil (1880-1896) y del Ayuntamiento de Pamplona (1897-1935).

No obstante, el dato a tener en cuenta para el período elegido es que más del $70 \%$ de los fallecimientos menores de 10 años se producen en los domicilios familiares. Para poder valorar en su justa medida la sobremortalidad en las viviendas particulares es necesario partir de algunos factores básicos: las carencias higiénico-sanitarias de las viviendas, el carácter be-

C. Gil, ante el aumento de partos de solteras en la Maternidad desde 1925 afirma: “las casas clandestinas para dar a luz donde por lo general no existe ni dirección técnica ni higiene muy depuradas, habrán visto considerablemente disminuida su clientela" (...) "Es muy posible que esta clase de establecimientos lo continuen perdiendo ya que como demuestran los numeros expuestos la Casa de Maternidad va ofreciendo de día en dia mayor confianza y seguridad a las mujeres solteras" (Memoria estadística de la Casa de Maternidad, pág. 4).

Uribe-Etxeberaia, A., Protección a la infancia y la maternidad, pág. 383. 
néfico y estigmatizante de los centros asistenciales, la escasez de instituciones de sanación, la limitada cultura higiénica de la sociedad, etc. Junto a ellos y no con menos importancia, se desarrolla en estos años una nueva valoración de la infancia y una nueva idea de la maternidad encaminadas a reducir los fallecimientos. Madre e hijo adquieren una nueva dimensión y, con ellos, la familia como célula de atención y salud.

Se discute y se perfila desde finales del siglo $x \mid x$ el modelo de mujermadre que se desea para España. Es una mujer con una clara dimensión higiénica y moral, y capacitada para acelerar la lucha contra la mortalidad y la morbilidad. Entre sus funciones sobresalen la administración del hogar, la defensa de la vida, el cuidado de la salud de los alli reunidos y "la propagación de la cultura social por medio de la educación del niño y el buen gobierno doméstico " ${ }^{8}$. Las campañas de vulgarización de los rudimentos más elementales de la pediatría y la profilaxis general, dirigidas a reducir la morbilidad y mortalidad de la población más joven, tendrán entre sus objetivos la promoción de lo que se entendía por «buenas madres».

"Sólo la madre que alimenta y cuida de su hijo, prodigándole todas las atenciones que su estado necesita, rodeándole de todo género de precauciones, viviendo su misma vida y no ocupándose de nada que de cualquier modo pueda perjudicar al nuevo sér, cumple la misión que la Naturaleza le confía y no abandona á su hijo. (...) El amor más grande, más puro, el más abnegado, el amor de madre, que solo sienten con viveza intensa las madres que, además de serlo, crian á sus hijos" ${ }^{9}$.

Las dos claves para obtener el éxito esperado de una mayor participación de la mujer-madre en cuestiones de salud serán la limpieza corporal y la alimentación de su hijo. Para la inmensa mayoría de la población de Pamplona, la limpieza corporal era el mejor vehiculo para contraer enfermedades tan letales como las bronquitis, pulmonías o neumonías. Frente a este desprecio se alzaron distintas voces como las de A. Lazcano (1903) que intentaban dar una valoración en positivo de esa práctica tan reprobada. Hablar de limpieza era hablar de nivel de vida, de educación, de integración social plena.

«El niño es el ser que más cuidados necesita de una casa; su limpieza es el quehacer único que debe embargar a toda madre; de ella y de su escrupulosa asiduidad resultará el día de mañana que pueda ser el báculo de su vejez" ${ }^{10}$.

Perdiguero, E., “Popularización de la higiene....", pág. 235.

González Revilla, G., La protección de...., págs. 44 y 46.

Diario de Navarra, 21-9-1910. 
A la luz de los abundantes artículos de prensa local preocupados por estos temas ${ }^{11}$, se comprende que sólo a través de la educación de grupos de mujeres fuera posible modificar los comportamientos higiénicos y alimenticios dirigidos a los menores de diez años ${ }^{12}$. Estas mujeres, no analfabetas, tendrían como misión "enseñar á las que no leen. (...) que el único medio de criar niños robustos es el uso continuo del baño" y que la mayoría de los procesos gastrointestinales estaban relacionados con una deficiente alimentación ya "sea por la mala calidad de las comidas ó ya por la falta de régimen de éstas, tal como las horas fijas en el comer" ${ }^{13}$. El baño regular y la lactancia materna eran los mejores instrumentos de lucha contra la enfermedad y la muerte de los más jóvenes. Estos dos factores se traducian para el conjunto de la población en limpieza personal y familiar y en alimentación regular y variada, siempre alejada del consumo habitual de alcohol y de alimentos en mal estado o adulterados.

Este sistema asistencial hacía recaer sobre las madres la responsabilidad de velar por la salud de sus hijos, siendo la familia y no las instituciones públicas, religiosas o privadas la encargada de la protección de la población más joven, especialmente de los menores de un año. Sólo cuando el núcleo familiar o los progenitores, en el caso de los niño/as, no pudieran hacerse cargo de parte de sus miembros entraba en funcionamiento la "caridad social», cuyas instituciones centrales de Pamplona son el Hospital Civil y la Inclusa.

"De la posición de la madre, de sus situación, de las circunstancias de su vida, depende el bien y el mal de sus hijos, su salud y su entermedad, la vida y la muerte, la suerte ó la desgracia» ${ }^{14}$.

Retomando el hilo conductor del cuadro 1 y descendiendo al detalle, hay que diferenciar dentro de las residencias particulares a aquellas calles con mayor letalidad de aquellas otras más saludables. Evidentemente el

"Sobre este punto hay más información en: S. ANAUT, "La infancia en Pamplona....", 1994.

12 Con la aparición de los consultorios de lactantes -comienzos del Novecientos- se introduce en España una práctica en la que el médico realiza consultas y diagnósticos colectivos de niños estando sus madres presentes. Por este sistema se daban también consejos higiénicos y alimenticios y se otorgaban diplomas de honor "a la buena madre". En los años treinta los Dispensarios realizaban conferencias semanales durante seis meses al año, además de algunos cursos en las escuelas municipales. Gracias a testimonios orales hemos podido confirmar este mismo funcionamiento en Pamplona -el principal responsable de ello fue el doctor S. Húder-, lo cual es un sintoma del alcance nacional de las propuestas higiénicas y sanitarias emanadas desde las correspondientes instancias oficiales.

13 Diario de Navarra, 21-9-1910 y 11-8-1906, respectivamente.

14 Gonzalez Revilla, G., Op. cit., pág. 37. 
pertenecer a uno u otro grupo de calles depende del carácter intrínseco de las mismas, es decir, aquellas calles más largas, situadas dentro de la muralla y con inmuebles de cinco o más plantas, con escasos vanos y mal iluminadas serán bastante más mortíferas que aquellas otras en las que no converjan todos estos factores. El resultado obtenido ha sido el siguiente: la calle Mayor concentró al 6,37\% en 1881 de los finados y en 1931 al 3,2\%, permaneciendo siempre en la cabeza del callejero de la ciudad. En este último año el barrio de extramuros de la Rochapea registró el porcentaje más elevado de todo el conjunto de la ciudad $-4,53 \%$ - mostrando con ello que es un barrio en plena expansión tras la supresión en los años veinte de las prohibiciones impuestas por el Ramo de Guerra para urbanizarlo y que las condiciones de vida de sus residentes eran mucho peores que las del resto de Pamplona.

Dentro del recinto amurallado, tras la calle Mayor, las de Descalzos, Estafeta y Carmen completan el panorama. Se hace necesario traer a colación aquí el hecho de que la calle Mayor y sus alrededores fueran calificados ya en el siglo XI como unas vías malolientes, sombrías, poco ventiladas y de trazado tortuoso, lo cual demuestra los pocos cambios que experimentaron a lo largo de su historia. Con el cambio de siglo se unen a estas calles otras como las de S. Nicolás, Jarauta o el barrio de S. Juan, alcanzando porcentajes similares o superiores a las de las tradicionales calles más mortíferas.

La verdadera aportación del cuadro 1 a nuestro objetivo de conocer el nivel de desarrollo del sistema asistencial de Pamplona hace referencia a los centros asistenciales y específicos. Los primeros verán aumentar de manera muy notable las defunciones en este medio siglo, en tanto que los segundos las verán retroceder hasta casi la desaparición. Los importantes avances en los campos de las infraestructuras - por ejemplo, con la construcción del Hospital de Barañain se levantaron una nueva Maternidad e Inclusa (1933) - , de las mejoras en la asistencia médica y pediátrica y un cambio en la concepción y percepción del sistema sanitario y asistencial, favorecieron ese lógico crecimiento de los óbitos, en particular en el Manicomio ${ }^{15}$, Hospital Civil y sus dependencias anejas. Esta evolución no fue a costa sólo de las residencias particulares, lo fue todavía más de otro centro: el Hospital Militar.

15 Las defunciones en el Manicomio aumentaron considerablemente en los dos años analizados del siglo xx. En 1906 fueron 22, de los cuales 16 eran varones, mientras en 1931 llegaron a los 52, de los que 35 también eran hombres. Por tanto la sobremortalidad masculina parece ser lo que define a esta institución, y según algunos comentarios periodísticos tiene que ver con el mayor consumo de alcohol de éstos. 
Los efectos de la tercera guerra carlista se dejaron sentir hasta el siglo $x x$ en cuanto a la presencia de un numeroso contingente militar en Pamplona y su Cuenca. Los militares, obviamente, serán atendidos en una institución particular que perderá su papel en la medida que se vaya reduciendo la población acuartelada y se vayan obteniendo triunfos sobre enfermedades infectocontagiosas como la fiebre tifoidea ${ }^{16}$, especialmente letales entre la población acuartelada. Estas pautas no son aplicables al sexo femenino, que experimenta algún cambio destacable únicamente en los centros asistenciales y ello parece estar asociado al ya apuntado incremento de ingresos de mujeres en la Casa de Maternidad.

Gracias al denodado esfuerzo de hombres como los doctores y concejales D. Carlos Gil, D. Cipriano Monzón, D. Agustín Lazcano, etc., de los numerosos y anónimos médicos y comadronas encargados de la asistencia domiciliaria e incluso de las propias instituciones municipales, se pudo lograr el aumento considerable de ingresos de mujeres gestantes en la Maternidad del Hospital en los años treinta. Atendiendo a las cifras recogidas por el doctor $\mathrm{C}$. Gil se pasó de 75 nacimientos en la sala de solteras en 1926 a 106 en 1930 con un "rendimiento del departamento durante el último año de un $24,24 \%$ mayor que en los cinco años anteriores" ${ }^{17}$. En esta década, pues, se percibe una creciente preferencia entre las mujeres con limitados recursos económicos por la asistencia controlada, al menos en el momento del parto, y por el abandono de los recién nacidos institucionalizado desde las instancias de la Maternidad hacia la Inclusa, en lugar de las tradicionales vías de la exposición o del torno. A la vez se intuye una suavización en el tema de la descalificación y la pérdida de honor femenino por alumbrar a una criatura no légitima ${ }^{18}$. Esta nueva imagen de la madre que asume plenamente su maternidad habla de la consumación del modelo burgués de la "buena madre" ${ }^{19}$.

Un breve comentario merecen los fetos que han quedado recogidos en el cuadro 1 bajo el epígrafe "s.sex.", aunque no supongan más de un 7\% del total de las defunciones anuales. Este grupo es quizá el que mejor refleja la evolución del sistema asistencial de Pamplona por cuanto atestigua que se duplican las defunciones en los centros asistenciales y sanitarios a costa de un fuerte descenso de los acontecidos en las residencias particulares. Por tanto, parece ser que en la capital navarra la población feme-

Alcanzaron las fiebres tifoideas al $11,2 \%$ de varones en 1881 y al $0,4 \%$ en 1931

GIL, C., Op. cit., pág. 3.

18 A partir de 1932 son cada vez más frecuentes los registros de defunciones de niños menores de un año - A.M.P. - en los que se especifica el nombre completo de la madre.

VALVEROE. L., Entre el deshonor..., pág. 153. 
nina está cada vez más concienciada de que debe dar a luz en las instituciones pertinentes y no dejarse llevar por parteras poco competentes y por la costumbre de quedarse en las casas a sabiendas de que no reunen las condiciones minimas para garantizar un parto sin complicaciones.

Esta conclusión conduce hacia dos nuevas reflexiones. La primera hace referencia a que el reformismo social imperante centró la mayor parte de sus esfuerzos en mejorar la asistencia benéfica dirigida a dos de los grupos más desprotegidos: las mujeres y los niños/as. La segunda reflexión plantea el carácter selectivo de las defunciones en las instituciones benéficas, es decir, la edad y la condición socio-económica son las variables que marcan la geografía de las defunciones.

La creciente generalización de la miseria en Pamplona, en particular en el cambio de siglo y en el tránsito de la década de los veinte a los treinta del siglo $x x$, no se correspondió con un incremento de las defunciones en los centros asistenciales dependientes del Ayuntamiento y/o de la Diputación Provincial, excepto para la población menor de diez años. En consecuencia, el sistema asistencial y de sanación en Pamplona no fue capaz de atender a las exigencias de una sociedad muy vulnerable ante el empobrecimiento y la pérdida de salud. Los problemas financieros del Consistorio serán exgrimidos para no atender dichas demandas sociales y para trasladar a la familia esa responsabilidad de asistencia.

Sólo quedaría por comentar el exiguo grupo de defunciones recogidas en el cuadro 1 bajo la denominación "sin especificar el lugar». La mayoría de los casos corresponden a decesos de recién nacidos o con menos de una semana de vida (gráfico 1). Bajo este epígrafe se han agrupado los casos en los que no se deja constancia del lugar del fallecimiento y aquellos otros en los que es posible hablar de abandono ante la muerte de las criaturas. Se han encontrado casos de niños muertos de muy corta edad en las alcantarillas, cementerio, en las carreteras o caminos próximos a la ciudad, en la estación de ferrocarril, etc. Estos abandonos constatan el problema de la marginación, el deshonor y la miseria de muchas madres, especialmente de las solteras. En los libros de defunciones del Registro Civil, hasta el cambio de siglo, se podian encontrar descripciones de este tipo de casos como los que siguen.

"El Sr. Juez de la misma (Pamplona) dispondrá la inscripción en el Registro Civil, de la defunción de una niña de cuatro á seis dias de existencia que apareció ó fue depositada en el torno de la Casa Inclusa de esta Provincia á las diez y media de la noche del 22 del corriente y que segun un papel que la acompañaba había fallecido á las 5 de la misma tarde á consecuencia de un catarro infantil" (24-3-1886). 
"El Sr Juez hace entrega de un mandamiento de dicho Juzgado para este Municipal en el que se ordena la inscripción de defunción de una criatura de sexo femenino; que ha sido hallada muerta luego de las ocho de la mañana de ayer, en la calleja de la calle Cuesta del Palacio, frente a la casa número 47 de dicha calle en el suelo de la misma frente á la pared posterior del Convento de Monjas de las Adoratrices, la que falleció a consecuencia de la falta de ligadura del cordón umbilical ó por asfixia por estrangulamiento" (...) "Que se ignora á quien pertenece la mencionada criatura, asi como tambien cual sea su procedencia" (4-10-1891).

Son dos ejemplos de los pocos que se han encontrado con una descripción tan detallada de los finados. En ellos el detonante principal del abandono es la muerte de la criatura. No debió haber, por tanto, intencionalidad previa del abandono a pesar de ser madres solteras, aunque en el fechado en 1891 la imprecisión a la hora de diagnosticar la causa de muerte puede conducir la reflexión hacia dos situaciones muy distintas: problemas durante el parto por falta de asistencia cualificada y el infanticidio por medio de "estrangulamiento" ${ }^{20}$. La estabilidad de las defunciones en este grupo a lo largo de todo el período objeto de estudio nos hace pensar en el descenso de la práctica del abandono y en la mejora de la calidad de las partidas de los Libros de Defunciones. Para el primer supuesto, es decir, la reducción del abandono fuera de las instituciones existentes, hay que observar que entre 1880 y 1934 el número de ilegítimos en la Inclusa y en la Casa de Maternidad no cesó de crecer hasta superar las tres cuartas partes del total de sus ingresos ${ }^{21}$. Esta evolución ascendente de los ingresos fue paralela a la de las defunciones de los menores de diez años en los centros asistenciales hasta 1933. En este cambio de actitud debió recaer la explicación de la estable curva de las defunciones "sin especificar el lugar" (gráfico 1). Es difícil recurrir a este elemento explicativo cuando se analiza el conjunto de las defunciones (cuadro 1). La mayor responsabilidad en este caso pasará a las variaciones impuestas por los registradores.

De lo hasta aquí expuesto se puede concluir que el sistema asistencial de Pamplona apenas si modificó sus funciones, objetivos y número de instituciones encargadas de cumplir con dicha misión social a lo largo de todo el período objeto de estudio. Las preocupaciones de los responsables del mismo sólo se centraron en la mejora de las instalaciones y en la ampliación de sus servicios. Los escasos avances que se produjeron hacia la

Puesto que nuestro objetivo no es explicar el abandono remitimios a los trabajos recogidos en la bibliografía de L. VALVERDE y A. URiBe-ETXEBERRIA.

Val.verde, L., Op. cit., págs. 81-85. 
modernización de dicho sistema no pudieron más que modificar lentamente la actitud de la población hacia él y favorecer el aumento relativo de los decesos de los grupos de población más vulnerables en estos centros en lugar de las residencias particulares, con lo que de mejora para la salud de la familia y de los vecinos ello conllevaba. En consecuencia, la sociedad de la capital navarra se hallaba claramente desasistida en un momento en el que los cambios en la vida económica exigían transformaciones en profundidad en la sociedad y en el papel desempeñado por los poderes públicos.

\section{LA INCLUSA: ENTRE LA VIDA Y LA MUERTE}

Dentro del tejido asistencial de Pamplona, una de las instituciones más importantes tanto para la capital como para la provincia fue la Inclusa. La Casa-Inclusa de Pamplona se creó en 1806 para socorrer a «las Mugeres mas miserables que fuera del camino real se anticipan a ser Madres, $y$ que no teniendo medios de subsistir tal vez malogran el precioso fruto que en si lleban ...precaviendo por este medio muchos infanticidios y haviendo al mismo tiempo de procurar la cura radical de esas infelices" ${ }^{22}$. Estas condiciones básicas para el ingreso de madres e hijos o de niños solos marcarán la evolución de la institución hacia lo que a partir de mediados del siglo XIX parece ser su misión central: la recepción de niños abandonados ilegítimos en su inmensa mayoría. Los motivos por los que se solicitaba el ingreso de los hijos eran: pobreza, fallecimiento de la madre, horfandad, nacimientos múltiples y ausencia de uno de lo cónyuges por trabajo o por ingreso en prisión u otra institución. De esta forma, la Inclusa se va convirtiendo en una institución de ayuda a la infancia en sus dos vertientes, la moral y la socioeconómica. Para la sociedad "no es sólo un sustituto de la nodriza; es también un depósito salvavidas que se nutre en gran medida de la marginalidad" ${ }^{23}$.

A partir de la segunda década del siglo xx fue completando sus servicios hasta asumir funciones y actividades como las de la Gota de leche, casa-cuna, asilo de párvulos, asilo de niñas adolescentes y mujeres adultas, y casa de maternidad gratuita y de pago. Como resultado, deja clara su inclinación preferente a los más pequeños y a la mujer, a la que tutela hasta el momento de contraer matrimonio o "salir a servir".

22 Valverde, L., Op.cit., pág. 53.

23 Valverde, L., Op.cit., pág. 76. 
La mayoría de los niños ingresados en esta institución proceden de la Maternidad y de otros medios (cuadro 2), de modo que en torno al $80 \%$ son niños ilegítimos cuyas probabilidades de supervivencia fuera de la Inclusa eran mínimas. Para gran parte de estos niños su abandono definitivo o temporal era la única tabla de salvación en una sociedad en la que no tenían un lugar. Esta posibilidad no era del todo real por las deficiencias que presentaba tanto en sus condiciones de habitabilidad como por la escasez de recursos humanos y económicos ${ }^{24}$. Como consecuencia de estos condicionantes externos y de la marginación social, tanto el Hospital como sus dependencias de Maternidad e Inclusa se convirtieron en "instituciones insalubres" y, por ello, "verdaderos focos de infección" peligrosos para la salud de la población de la ciudad y de los mismos ingresados ${ }^{25}$.

Cuadro 2: Modalidades de ingreso en la Inclusa de Pamplona ${ }^{26}(\%)$.

\begin{tabular}{cccc}
\hline AÑO & LEG. & H. Y MAT & OTROS \\
\hline $1880-84$ & 9,8 & 36,7 & 53,5 \\
$1900-04$ & 12 & 51,1 & 36,9 \\
$1910-14$ & 9,3 & 57,2 & 33,5 \\
$1920-24$ & 11,6 & 47,1 & 41,3 \\
$1930-35$ & 15,9 & 54,5 & 29,6 \\
\hline
\end{tabular}

FUENTE: A. Uribe-Exeberria (1993: 560).

Es la mortalidad que se producía en la Inclusa la que despertó los mayores temores sobre de los niveles de salud dentro y fuera de la misma. A. Uribe-Etxeberría ha recogido el número de ingresos y fallecidos en la Inclusa entre 1890 y $1930^{27}$. A la conclusión a la que llega es a que en la

\footnotetext{
24 Mucho más radical en sus afirmaciones que el navarro C. GIL es J. Bravo Frias por lo que es muy probable que las condiciones en que se hallaban otras inclusas en España fueran mucho peores que las de Pamplona: "Es tal la rutina que se sigue en la admisión y salida de los niños; es tal la práctica que con el tiempo han adquirido las hermanas de la Caridad en el trato de los niño, a la antigua, y tan pocos los remedios que el médico puede allegar para el tratamiento y mejora de las condiciones en que viven los asilados, que el papel del médico no puede ser más desairado de lo que es" (..) "El personal técnico auxiliar siempre ha sido escaso; ordinariamente ha tomado aquello como un cantón en el que no hay nada que hacer. (BRAvo, J., La transformación...., págs. 30-32).

25 Huder, S., Desarrollo de Pamplona...., pág. 38.

26 Se han agrupado las diferentes modalidad en: legítimos (Leg.), Hospital y Maternidad (H. y Mat) y por torno, abandono y exposición (Otros).

${ }_{27}$ URIBE-ETXEBerRiA, A., Op. cit., pág. 399.
} 
última década del siglo XIX y entre 1909-14 sobrevivieron en la Inclusa casi el $50 \%$ de los ingresados de todas las edades, en tanto que durante el resto del primer tercio del siglo xx ronda el $40 \%{ }^{28}$. El grupo de edad más afectado por esta sobremortalidad es el menor de un año. En estas circunstancias no hay que olvidar que los «niños a lactar (fuera de la Inclusa) son los más robustos de los que se encuentran en la Institución, y disponen de una nodriza cada uno de ellos mientras que los que permanecen en la Casa reciben una alimentación artificial o mixta" ${ }^{29}$. El doctor C. Gil pone el dedo en la llaga al afirmar que la mortalidad excesiva de la Inclusa está producida por una deficiente alimentación y atención a los niños, niños cuyas probabilidades de supervivencia fuera de la misma son muy superiores a los que permanecen en ella.

Cotejadas nuestras cifras absolutas, obtenidas a partir de los libros de defunciones del Ayuntamiento de Pamplona y del Registro Civil, con las aportadas por A. Uribe-Etxeberría se concluye que estas fuentes oficiales subregistraban de manera notoria los decesos de la Inclusa. Este subregistro puede estar condicionado por las salidas a lactar a otras localidades, cuyos niños/as fallecían en la localidad de residencia de la nodriza por lo que no siempre eran registrados en Pamplona, e igualmente por cierta desidia, intencionada o no, que llevaba a ocultar la dramática situación de los niños de la Casa ante la sociedad pamplonesa.

En un primer acercamiento a las cifras del cuadro 3 hay que valorar que las defunciones en la Inclusa pasaron de representar el $4,8 \%$ del total de las defunciones de la ciudad en 1881 a casi el $9 \%$ en 1931, y del 25 al $30 \%$ del total de las defunciones de los menores de diez años de las últimas décadas del siglo XIX a más del $40 \%$ en los años veinte y treinta del presente siglo, con la salvedad de los años 1934 y 1935. Si esta información se desglosa por subgrupos de edad, se observa que los fallecimientos infantiles en la Inclusa llegaron a representar, en concreto en el siglo xx, más del $30 \%$ de las defunciones infantiles totales. Por tanto, si la mortalidad infantil de Pamplona en su conjunto es un problema social y médico de gran magnitud, lo es más cuando se comprueba su concentración en una única institución. En otras palabras, para que se hiciera realidad el descenso de la mortalidad infantil en Pamplona era imprescindible transformar la Inclusa y, por ello, actuar sobre las causas que conducían al ingreso de los niños en la Institución.

28 J. Bravo Frias alude a niveles de mortalidad muy superiores a los de Pamplona: "En la lucha entablada contra la mortalidad infantil pronto se vio que la de las Inclusas era verdaderamente horrorosa, llegando a alcanzar la cifra de 70 a $80 \%$ de los ingresados, y por tanto. en vez de ser verdaderos viveros humanos, eran Necropolis infantiles" (Op. cit., 8).

29 GIL, C., Op. cit., pág. 8. 
Cuadro 3: Distribución porcentual de las defunciones de la Inclusa por grupos de edad respecto del total de las defunciones de esos mismos grupos de edad (\%)

\begin{tabular}{|c|c|c|c|c|c|c|c|}
\hline AÑO & INF. & JUV. 1.4 & JUV. 5.10 & AÑO & INF. & JUV. 1.4 & JUV. 5.10 \\
\hline 1880 & 14,1 & 7,4 & 7,5 & 1908 & 27,3 & 4,6 & 0 \\
\hline 1881 & 18,3 & 2,9 & 2,6 & 1909 & 22,9 & 2,7 & 3,6 \\
\hline 1882 & 18,6 & 3,8 & 2 & 1910 & 44,7 & 6,5 & 3,4 \\
\hline 1883 & 26,7 & 3,9 & 1,2 & 1911 & 51,1 & 10,1 & 2,3 \\
\hline 1884 & 28,6 & 5,9 & 2,1 & 1912 & 52,3 & 16,3 & 0 \\
\hline 1885 & 25,6 & 9,2 & 6,1 & 1913 & 53,5 & 8,8 & 0 \\
\hline 1886 & 28,5 & 4,2 & 3 & 1914 & 49,7 & 11,1 & 0 \\
\hline 1887 & 17,5 & 2,0 & 1,8 & 1915 & 17,6 & 8,6 & 0 \\
\hline 1888 & 11,9 & 2,8 & 3,1 & 1916 & 34,0 & 8,6 & 0 \\
\hline 1889 & 11,9 & 7,1 & 9,8 & 1917 & 39,6 & 30,5 & 0 \\
\hline 1890 & 12,6 & 7,2 & 7,4 & 1918 & 45,8 & 21,6 & 6,3 \\
\hline 1891 & 14,7 & 5,4 & 10 & 1919 & 32,7 & 9,3 & 0 \\
\hline 1892 & 18,7 & 6,1 & 7,7 & 1920 & 35,3 & 2,6 & 8,6 \\
\hline 1893 & 15,7 & 9,4 & 0 & 1921 & 46,3 & 6,8 & 0 \\
\hline 1894 & 18,5 & 2,8 & 2,0 & 1922 & 45,9 & 19,7 & 0 \\
\hline 1895 & 9,6 & 11,5 & 2,1 & 1923 & 23,2 & 7,2 & 7,7 \\
\hline 1896 & 16,9 & 4,4 & 0 & 1924 & 37,9 & 10,2 & 0 \\
\hline 1897 & 24,1 & 6,4 & 17,9 & 1925 & 38,4 & 8,9 & 0 \\
\hline 1898 & 20,8 & 8,3 & 2,9 & 1926 & 41,7 & 8,1 & 0 \\
\hline 1899 & 21,5 & 3,3 & 0 & 1927 & 27,3 & 16,1 & 0 \\
\hline 1900 & 23,7 & 6,8 & 1,7 & 1928 & 44,8 & 10,0 & 0 \\
\hline 1901 & 30,2 & 5,2 & 2,4 & 1929 & 35,3 & 11,8 & 4,0 \\
\hline 1902 & 39,5 & 7,9 & 12,5 & 1930 & 42,3 & 8,1 & 4,8 \\
\hline 1903 & 32,7 & 1,4 & 0 & 1931 & 35,7 & 12,5 & 0 \\
\hline 1904 & 27,0 & 2,0 & 2,8 & 1932 & 31,9 & 6,5 & 9,5 \\
\hline 1905 & 43,1 & 8,0 & 0 & 1933 & 37,4 & 5,2 & 0 \\
\hline 1906 & 28,6 & 4,9 & 7,0 & 1934 & 22,0 & 15,5 & 0 \\
\hline 1907 & 36,8 & 13,3 & 2,9 & 1935 & 6,2 & 2,3 & 0 \\
\hline
\end{tabular}

FUENTE: Elaboración propia a partir de los libros de defunciones del Ayuntamiento de Pamplona y del Registro Civil.

Ante la imposibilidad de descartar tanto de los ingresos como de las defunciones los ingresados no nacidos o que no debieron nacer en Pamplona, sobre todo por la falta de información sobre los progenitores y 
porque en las partidas de defunción son casos aislados los registrados como de fuera de la capital, se ha procedido a realizar un ejercicio muy discutible, pero a la vez muy ilustrativo y significativo. Se ha calculado la probabilidad de morir antes de cumplir el primer aniversario estimando (q0) y destimando (q0-inc) los decesos de la Inclusa. El resultado ha quedado recogido en el gráfico 2.

Gráfico 2: Probabilidad de morir antes de alcanzar el primer aniversario incluyendo (q0) y desestimando (qo-inc) los decesos producidos en la Inclusa de Pamplona ${ }^{30}$



FUENTE: Elaboración propia a partir de los libros de defunciones del Registro Civil y del Ayuntamiento de Pamplona.

El trazado que describen ambas curvas permite llegar a conclusiones muy significativas. La primera y ya apuntada anteriormente, el creciente peso de los finados infantiles de la Inclusa respecto del total infantil a partir del cambio de siglo, cuando ambas curvas tienden a separarse. Esta progresiva importancia es un síntoma del nuevo papel que deberán cumplir los centros asistenciales con el paso del tiempo, es decir, la asistencia y sanación desde instituciones tuteladas por los poderes públicos no sólo tendrá que atender a los sectores sociales más depauperados y marginados, también deberá dar respuesta a nuevos sectores y nuevas demandas que a la postre mejoren los niveles de salud colectiva - un ejemplo ya reseñado fue la aparición del departamento de casadas de la Maternidad-. 
En segundo lugar, las coyunturas críticas del cambio de siglo y de la epidemia gripal no dejaron huella reseñable sobre las defunciones de los menores de diez años acaecidas fuera de la Inclusa. Así, entre finales de los años noventa y los primeros años del siglo xx, el aumento de las defunciones infantiles estará bajo la influencia de un comportamiento específico ligado a la crisis agraria de esos años. En una ciudad sin capacidad para cubrir las demandas laborales y de infraestructuras que se suscitaron a partir de la llegada de numerosos trabajadores del campo, éstos, ante la presión de la pobreza, se verán abocados a abandonar a sus hijos allí donde se les podía dar una oportunidad. Por su parte, la epidemia gripal es lógico que actuara con mayor virulencia en la Inclusa porque en ella confluyen factores de alto riesgo para la salud: hacinamiento, falta de aislamiento de los afectados y debilidad fisiológica de los niños. Como se recoge en el cuadro 3 , el $46 \%$ de las defunciones infantiles y el $28 \%$ de las juveniles en 1918 tuvieron lugar en esta institución ${ }^{31}$. Con estos datos es fácil entender que la Inclusa fuera considerada un foco de infección muy peligroso para la población ${ }^{32}$.

Conviene destacar, en tercer lugar, el cambio de tendencia en los años treinta de la probabilidad de morir sin incluir los finados de la Inclusa (gráfico 2). Las únicas razones que encontramos para explicar este giro tan radical son las mejoras de habitabilidad al trasladarse al nuevo recinto en Barañain y en las atenciones sanitarias y de la alimentación e higiene de la Inclusa, y el empeoramiento de las condiciones de vida de la población. De este modo, el descenso de las defunciones en la institución no tuvo su correlato en otro proceso similar fuera de la misma.

Por último, la tendencia general de las curvas q0-inc y q0 del gráfico 2 nos permite confirmar que, frente a la estabilidad de la mortalidad infantil total (q0), la mortalidad infantil sin los óbitos de la Inclusa (q0-inc) marca una tendencia claramente descendente hasta 1912-14, habiéndose acelerado al iniciarse el siglo xx y cerrándose el periodo 1880-1914 con una reducción de hasta la mitad de la probabilidad de morir. Entre 1914 y 1919 se quiebra el declive apuntado, pero ya no se recuperan los niveles de finales de la primera década del siglo xx. Hasta aquí este comportamiento sigue el mismo patrón que el de la probabilidad de morir de uno a cuatro años. Ahora bien, en los años treinta se produce una ruptura con el patrón que había ido describiendo hasta entonces. Esta ruptura no es explicable

\footnotetext{
En octubre de 1918 fallecieron el $160,2 \%$ de los internos y en noviembre el $105,6 \%$ (Boletín Mensual de Estadistica Municipal de Pamplona).

32 Huder, S., Op. cit., pág. 38.
} 
más que por la importancia, como agente perturbador de la evolución de una población, de los flujos inmigratorios. Es posible, en suma, concluir que tanto la Inclusa como los movimientos inmigratorios determinaron el modelo de modernización de la mortalidad en Pamplona y, por tanto, fueron delimitando las exigencias de un cambio en la rígida red asistencial.

Si a continuación desagregamos las defunciones de los menores de un año por subgrupos de edad también podemos encontrar algunas diferencias con respecto a la mortalidad infantil general (gráficos 2 y 3 ). Los óbitos perinatales ${ }^{33}$ tendieron a perder importancia relativa respecto al resto de los infantiles entre otras razones por la mejora de la atención obstétrica y ginecológica que se fue dando dentro de la institución al amparo de una legislación y una valoración del niño que exigía también la protección de la futura madre:

"Debemos pensar que la existencia del recién nacido, no tiene lugar en el momento mismo del parto sino en aquel instante en que se ha efectuado la fecundación. (...) Asi es en efecto, todas las vicisitudes y trastornos del organismo materno durante el embarazo repercuten infaliblemente sobre la viabilidad del nuevo ser. Debemos por tanto admitir como una ver-

Gráfico 3: Distribución proporcional de las defunciones infantiles de la Inclusa por subgrupos de edad (\%).

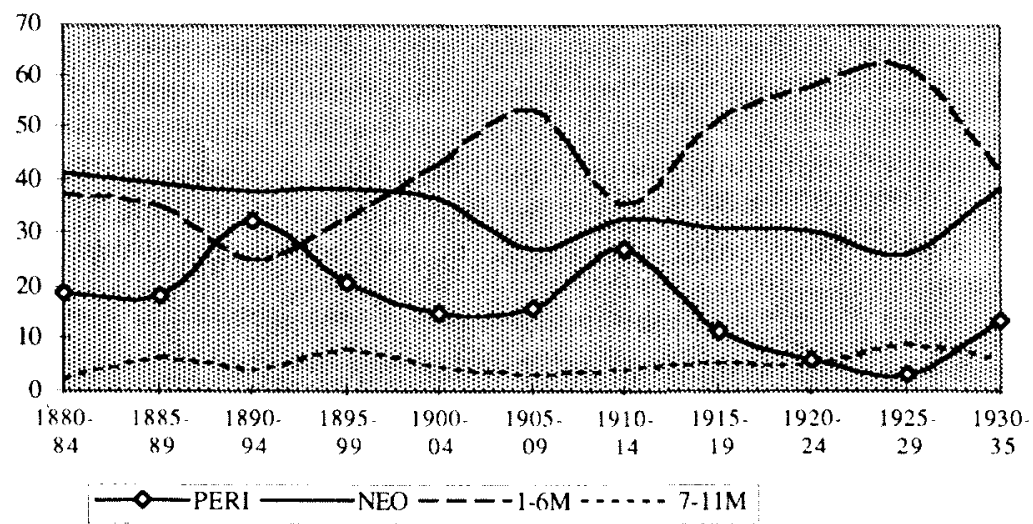

FUENTE: Elaboración propia a partir de los libros de defunciones del Registro Civil y del Ayuntamiento de Pamplona.

Entendemos por finados perinatales aquellos fallecidos antes de alcanzar con vida las primeras veinticuatro horas. 
dad axiomática, que la protección al niño debe empezar con anterioridad a la época del nacimiento" ${ }^{34}$.

Entre tanto, las defunciones neonatales -no incluyen las perinatalesy postneonatales de 7 a 11 meses mantuvieron una especial estabilidad a lo largo de todo el período (gráfico 3). Los finados postneonatales de 1 a 6 meses rompen la tendencia del resto de los subgrupos al concentrar a partir del inicio del siglo $x x$ entre el 40 y el $60 \%$ de las defunciones infantiles. Es un momento crítico por cuanto coincide con el inicio de la dentición y de una alimentación mixta ${ }^{35}$, que como es de suponer se iniciaba pronto en la Inclusa por falta de recursos económicos para contratar nodrizas y para mantener lactancias muy prolongadas. Del mismo gráfico, por último, se extrae como dato a tener en cuenta que es durante el primer mes - mortalidad neonatal estricta - cuando se produce el mayor número de decesos hasta el inicio de la nueva centuria y que desde entonces las defunciones de uno a once meses se imponen.

Analizada la mortalidad infantil en la Inclusa quedarian por comentar los fallecimientos juveniles que en ella se producen ${ }^{36}$. Hasta el quinquenio 1920-24 las defunciones de uno a cuatro años suelen superar el $15 \%$ del total de las ocurridas en la Inclusa, pasando desde esa fecha a proporciones menores al $8 \%$. Esta progresiva pérdida de relevancia de las edades juveniles es más notoria en los casos de cinco a diez años, ya que a partir del siglo $x x$ no superan el $1,5 \%$ del total. De las defunciones juveniles son las producidas con uno y dos años las más numerosas. Este subgrupo llega a sobrepasar el $80 \%$ de las defunciones de uno a cuatro años y el $65 \%$ del total de las juveniles de la Inclusa. Es durante este período de la vida, cuando los niños se hallan en una etapa crítica al coincidir la dentición con el inicio del destete progresivo, una mayor morbilidad y mortalidad por enfermedades infecciosas como el sarampión y una elevada vulnerabilidad ante las condiciones medioambientales.

Respesto a la intensidad de la mortalidad de los menores de diez años en la Inclusa a lo largo del año (gráfico 4), son los meses estivales de julio y agosto, seguidos de los meses otoñales, los que concentran la mayor proporción de defunciones. La importancia de las dolencias digestivas y

\footnotetext{
GiL, C., Maternidad. pág. 13.

Al utilizar el término alimentación mixta nos referimos tanto a la lactancia con leche materna y biberón como a la introducción en la alimentación de estos niños de alimentos no lácteos.

36 Hemos incluido en el cuadro 3 a los finados mayores de siete años por encontrar algunos casos aislados, ya que to habitual era que a partir de los siete años pasaran los supervivientes a la Casa de Misericordia o a familias.
} 
Gráfico 4: Ciclo anual de las defunciones de la Inclusa por quiquenios (\%).



FUENTE: Elaboración propia a partir de los libros de defunciones del Registro Civil y del Ayuntamiento de Pamplona.

respiratorias serán las causantes de tal reparto. La calidad y cantidad de los alimentos, las deficiencias del propio establecimiento, las características climáticas de la ciudad, el hacinamiento y la ausencia "de un local adecuado en donde puedan aislarse los niños convenientemente en casos de epidemia' ${ }^{37}$, explican la concentración de los decesos en el segundo semestre del año y, sobre todo, del mes de agosto. Como sucediera en la mortalidad infantil general, el quinquenio 1930-35 cuenta con indicios de una cierta modernización del modelo estacional de las defunciones, es decir, agosto pierde la hegemonía en favor de julio y diciembre, en tanto que febrero y marzo marcan alzas en el número de decesos, pudiendo así hablar de un cambio paulatino hacia un modelo moderno de transición de la mortalidad.

Para valorar en su justa medida la intensidad de la mortalidad a lo largo del año vamos a recurrir a las causas de muerte más representativas en esta Institución ${ }^{38}$. Con ello queremos insistir en los graves problemas de salud de los ingresados y en los avances sobre el control de las enfermedades infecciosas. Dejando a un lado los registros en los que no aparece la causa de defunción y cuya proporción en algunos años superó el 15\%, hasta el quinquenio 1925-29 predominaron las enfermedades infecciosas entre las que

Git, C., Memoria estadistica...., pág. 2.

Se han agrupado las enfermedades siguiendo la Clasificación abreviada de T. MCKEONW y la Clasificación Internacional de BERTILLON, tal y como se propone en J. BERNABEU, A. SANZ y otros (1996). 
sobresalen la sifilis, la bronquitis, la diarrea-enteritis, la tuberculosis y la meningitis. La importancia a finales del siglo $x \mid x$ de la sífilis justificará la preocupación de políticos, médicos e higienistas sobre la falta de moral de la sociedad a lo largo del primer tercio del Novecientos ${ }^{39}$. Por los testimonios recogidos en la prensa, no parece que hubiera muchos cambios en los comportamiento sociales, quizá la reducción del contingente militar en la ciudad, los progresivos mayores controles sobre la práctica de la prostitución y, sobre todo, los avances médicos ayudaron a ese importante descenso.

"(La sifilis) es el responsable en un 60\% de los casos de aquellos que nacen muertos, bien sea de término, o bien antes de terminar la gestación. Esta enfermedad que durante tantos años ha constituido un azote social más temible que la tuberculosis, lleva en la actualidad camino de desaparecer. (...) Si existe la enfermedad, con un tratamiento adecuado, se consigue el nacimiento de niños sanos" ${ }^{40}$.

La segunda causa de muerte en importancia es la diarrea-enteritis que suele cursar especialmente en los meses estivales y que está ligada a una alimentación deficiente. Deficiencia que en la Inclusa es considerable por cuanto no hay esmero a la hora de preparar los alimentos y la lactancia no suele ser materna/nodriza con plenas garantías. Su descenso por debajo del $8 \%$ no se produce hasta el sexenio 1930-35. Este declive coincide con los primeros síntomas de modernización del ya apuntado patrón estacional de las defunciones.

Por último, son las bronquitis, bronconeumonías y tuberculosis pulmonares las que constituyen el tercer grupo de enfermedades infecciosas altamente letales en la Inclusa. Sus evoluciones fueron muy diferentes por cuanto la tuberculosis solía ser consecuencia del contagio de la madre o nodriza, en tanto que las bronquitis y las bronconeumonías estaban asociadas a las condiciones de vida en el Centro y a la debilidad fisiológica de los niños. Ahora bien, mientras las bronquitis experimentan una caída muy importante tras un período álgido entre 1905 y 1924, las bronconeumonías no aparecen diagnosticadas como causas de muerte hasta el inicio del siglo $\mathrm{xx}$, si bien desde ese instante su crecimiento no cesará hasta experimentar un ascenso muy brusco en el sexenio 1930-35. Por tanto, es durante este periodo cuando las dolencias digestivas van dejando paso a las respiratorias, y este signo de cambio debió coincidir con la progresiva sustitución de las enfermedades infecciosas por las no infecciosas.

39 En 1885-89 alcanzó el 20,1\% frente al 1,3\% de 1930-35.

Ald GiL, C., Maternidad, pág. 17. 
A partir del quinquenio 1925-29 este signo del inicio de la transición epidemiológica en la Inclusa empieza a hacerse patente gracias al enorme peso que estaban adquiriendo los "vicios de conformación" - $37 \%$ Este tipo de diagnósticos se emplean en las defunciones de las edades más tempranas por lo que la mortalidad infantil que aqui se ha descrito justifica esta expresión diagnóstica, sin olvidar las variaciones en las preferencias por unos u otros diagnósticos dependiendo de las corrientes médicas imperantes ${ }^{41}$.

En consecuencia y a modo de síntesis, la elevada mortalidad infantil y juvenil y, por tanto, las escasas probabilidades de supervivencia de los ingresados en la Inclusa, aunque mayores que en otras del país, planteará la necesidad de adecuación y modernización de esta Institución. Se proponía transformarla en un centro de protección y sanación de los niños más indefensos. Sin embargo, esta demanda de la propia sociedad y de los facultativos quedó integrada en un discurso político-demográfico obcecado por las cuestiones presupuestarias y en un discurso higienista que parecía más preocupado por los efectos que sobre la moral y la salud colectiva pudiera tener esta sobremortalidad, que por los problemas de fondo de la Institución y del conjunto de la sociedad. Quizá por ello no es extraño que no se actuara antes sobre las instituciones asistenciales. De esta forma, pobreza e ilegitimidad componen el dúo de causas que conducen a la muerte en una mayor proporción a la población menor de diez años, especialmente a la población infantil.

"iLos pobres niños, muriéndose, solucionan por si solos infinidad de conflictos de orden económico; si vivieran exigirian un gasto que hoy no originan!, 42 .

La tendencia a aumentar la intervención del Estado y de las autoridades municipales en todo lo concerniente a los estados de salud, vida y muerte de las mujeres en edad de procrear y de los más pequeños se percibe igualmente a través del estudio de la evolución de las defunciones en la Inclusa. Sus efectos, no obstante, no parecen dar sus frutos hasta la década de los veinte y treinta. En suma, habrá que reiterar que la población de Pamplona y, en concreto, la menor de diez años se hallaba en estas fechas desasistida.

41 Dentro de los vicios de conformación el grupo más importante a partir de esas fechas corresponde a los clasificados por BERTILLON como "debilidad congénita. ictericia y escleremia" -categoria 10-. Es posible que antiguos diagnósticos de sífilis atendiendo a las consecuencias que apuntaba $C$. Git, pasen a esta rúbrica.

4. Bravo Frías, J., op. cit., pág. 4. 


\section{A MODO DE REFLEXIÓN FINAL}

La lucha por la supervivencia y la lucha por diseñar unos nuevos rumbos y valores para la vida de la ciudad entre 1880 y 1935 van a convivir en un equilibrio muy inestable que, sin llegar a causar grandes altercados de orden público ni fuertes polémicas, irá dando el triunfo a quienes defendian la modernización de Pamplona y su conversión en un espacio con un modo de vida urbano liberal-conservador, en definitiva, una ciudad de progreso económico y demográfico, de orden y pulcritud. El reto que se abría para las instancias municipales superaba su capacidad de respuesta rápida y eficiente, por lo que tendrá que dejar escapar de su control parcelas importantes como ciertos servicios asistenciales, la realización de proyectos urbanísticos concretos, el abastecimiento de agua, la fiscalización de gran parte de las actividades económicas, etc., muchas de ellas francamente rentables. A pesar de todo ello, es notorio el creciente intervencionismo municipal en la regulación de la vida urbana. Intervencionismo que va desde las ordenanzas referidas a la construcción de las nuevas viviendas a la política de saneamiento "físico y moral", pasando por una amplia gama de facetas asitenciales y de control sobre la vida económica de la población — por ejemplo, los «trabajos de invierno"- Todo ello forma parte de un proceso de cambio urbano y social que dejará su impronta en la demografía a través de sus efectos sobre la reducción de la mortalidad.

Los principales receptores de este intervencionismo municipal serán aquellos colectivos con mayor riesgo de pauperización: la infancia, la primera juventud, la mujer y los ancianos de las clases populares. Con todo, el conjunto, a veces inconexo, de la red de servicios sociales que se fue consolidando en estos años dejó en evidencia su capacidad para atender a una demanda creciente que, entre otras cosas, reclamaba una evolución más rápida de aquellos establecimientos con mayores rémoras del pasado - Hospital, Inclusa, etc.- - Gran parte de la debilidad de esta red se fundará en la ausencia de un beneficencia de alcance nacional, en las discrepancias entre las autoridades provinciales y municipales a la hora de delimitar sus atribuciones, en los limitados recursos financieros del Ayuntamiento y en la escasa flexibilidad para adaptarse a las nuevas exigencias de la sociedad. El panorama resultante es poco halagüeño, ya que la mayor parte de la población de Pamplona contaba con unas economias familiares en el límite subceptible de la pauperización, y hacia ellos no van encaminadas las instituciones y servicios asistenciales, de ahí que podamos afirmar sin lugar a dudas que la población de la capital navarra se hallaba desasistida. 
Las deficiencias en la administración e infraestructuas sanitarias se deben enmarcar en un proceso en el que la respuesta institucional y privada al problema de la miseria va desligándose muy lentamente de la tradicional caridad cristiana, y en una etapa en la historia de España en la que están triunfando los planteamientos regeneracionistas. La acción asistencial no hará más que aliviar las situaciones más dramáticas, pero no llegará a entrar en los problemas de fondo. La miseria, el pauperismo, es el estado socio-económico más generalizado e inestable en capitales de provincia como Pamplona, aunque no se pueda calificar de plaga, como se hace desde cierta prensa conservadora navarra ${ }^{43}$, sino más bien de un fenómeno que va a adquirir envergadura por la escasez de oportunidades laborales, por falta de renovación del sistema asistencial heredado, por ineficacia de una política urbanística clara o de una intervención más decidida de las autoridades en asuntos tan claves como el precio de los alquileres de las viviendas.

Desempleo, subalimentación, analfabetismo y desprotección componen los cuatro pilares sobre los que pivotan el nivel de salud y la evolución de una población como Pamplona en el período de la transición de su mortalidad. Para subsanar parcialmente estos desequilibrios intrínsecos del medio social urbano nacen respuestas de talante benéfico "que refuerzan la idea de una ciudad productiva, ordenada y segura" ( $\mathrm{P}$. Carasa Soto, 1994: 31), y nuevas actitudes desde instancias nacionales que, al margen de conservar la idea de que el pauperismo es "fuente de peligros sociales" y "amenaza constante para la salud y seguridad de las poblaciones", comienzan a hablar de prevención, de justicia social y solidaridad ${ }^{44}$. Esta sociedad urbana y burguesa irá infundiendo a través de su política social una serie de valores totalmente ajenos a las clases populares. Entre estos valores sobresalen el trabajo, el ahorro, la previsión, el orden, la obediencia política y el cuidado de la salud. Todos ellos quedaron perfectamente consolidados y difundidos por distintas instituciones y servicios, como la Inclusa, la Casa de Misericordia o la Gota de Leche.

En suma, el lugar registrado como aquel en el que se produce el fallecimiento informa sobre las costumbres y sus variaciones a lo largo del tiem-

43 "El pauperismo es una plaga de la sociedad moderna. su forma más repugnante es la mendicidad de oficio. En otros tiempos el señor feudal cuidaba de la alimentación de sus vasallos (...). La descentralización y el individualismo crearon millares y millares de pobres y la vagancia y los vicios coronaron la obra creando ejércitos de personas que viven á costa de la caridad de los demás hombres" (Diario de Navarra, 24-9-1904).

44 "Proyecto de Ley sobre Asistencia pública", Gaceta, 3-6-1916, Ministerio de Gobernación. 27-9-1919, pág. LIV. 
po en el tema de la muerte, sobre las diferencias socioeconómicas - a la Inclusa van a parar los hijos de la población marginada y empobrecida-y sobre el grado de desarrollo del sistema de asistencia y sanación de una población.

\section{BIBLIOGRAFÍA}

ANAIJT BRAvo, S., "La infancia en Pamplona durante el primer tercio del siglo XX", en revista Estudios de Ciencias Sociales, U.N.E.D., Pamplona, 1994.

Bernabeu, J.; Sanz, A.; Ramiro, D. y Robles, E., "De expresiones diagnósticas a causas de muerte: aspectos epidemiológicos en la transición de la mortalidad infantil y juvenil de la población rural española, 1841-1960", en Third Innocenti Seminar on the Decline of Infant and Child Mortality in Europe, Florencia, 1996.

Bravo Frias, J. y Alonso Muñoyerro, J., La transformación de las Inclusas, Imp. de M. Anguiano, Madrid, 1921.

Gıl.. C., Memoria estadística de la Casa de Maternidad y Expósitos de Navarra, Pamplona, 1930.

GiL, C., La Maternidad, Pamplona, 1930.

GonzAlez Revilla, G., La protección de la infancia abandonada (abandono y criminalidad de los niños), Imprenta de la Sociedad Anónima, 1907.

Huder, S., Desarrollo de Pamplona en los últimos cien años, Pamplona, 1935.

Lazcano, A., Higiene y salubridad públicas, Pamplona, 1903.

MONzón, C., La mortalidad infantil en Pamplona, 1903.

PErdiguero, E., "Popularización de la higiene en los manuales de economia doméstica en el tránsito de los siglos xix y xx", en BARONA, J. L., Malaltia i cultura, Trobades, Seminari d'Estudis sobre la Ciència, Valencia, 1995.

Uribe-Etxeberria Flores. A., Protección a la infancia y la maternidad. La Casa de Maternidad y Expósitos de Navarra (1890-1930), Tesis doctoral, U.P.V., 1993.

URIBE-ETXEBERRIA FLORES, A., Marginalidad protegida: mujeres y niños abandonados en Navarra (1890-1930), U.P.V., Bilbao, 1996.

VALVERDE, L., Entre el deshonor y la miseria. Infancia abandonada en Guipúzcoa y Navarra, ss. xVIII-xix, U.P.V., Bilbao, 1994. 